Theophilus Lazarus

Emory University,

Atlanta, GA, United States;

Prof T Lazarus Inc., Neuropsychology Practice,

Durban, South Africa

\title{
SARS-CoV-2 infection and neuropsychological outcomes
}

Abstract. The entire world is currently confronted with the Severe Acute Respiratory Syndrome Coronavirus 2 (SARS-CoV-2), a novel betacoronavirus causing the deadly pandemic of coronavirus disease 2019 (COVID-19). Since there is now increasing reports of neurological and cognitive problems, the impact of COVID-19 on neuropsychological functioning is unknown but is likely to leave residual problems.

Keywords: Severe Acute Respiratory Syndrome Coronavirus 2 (SARSCoV-2); Coronavirus Disease COVID-19; hypoxia; neurological; neuropsychological; cognitive; behaviour.

Introduction. The last pandemic was documented about a century ago (1918). Following that pandemic, Parkinson's Disease emerged as a major neurological disease that posed significant neurological and neuropsychological morbidity until the discovery of first-generation anti-Parkinsonian medication in the late 1940's, providing management of the disease. In the current pandemic, patients with comorbid health problems of all ages are at risk for serious health problems or sadly, have succumbed to COVID-19. Isolated case studies of pregnant women such as that of Hosier et al. [1] recently reported two cases suggesting that the SARS-CoV-2 virus may cross the placental barrier, infecting the fetus. IN Texas' Nucces County in the USA, 85 infants under the age of 1 year have tested positive for the novel coronavirus. In adults in particular, the impact of the virus has been varied with acute changes in breathing and hypoxia to blood clots and strokes, and microbleeds [2], in many instances, requiring ICU management and ventilation. The delayed effects of COVID-19 on neuropsychological functioning are unknown. Although the significant death-rate worldwide has triggered alarm and 
anxiety as well as fear of impending death particularly in the absence of a vaccine, psychological distress and other cerebral effects are reported. The impact on cerebral functioning is anticipated in view of two possible effects. Since SARS-CoV-2 uses the angiotensin converting enzyme 2 (ACE2) receptor [3] as an access portal to the lungs, the indirect effects of low blood oxygen saturation levels or hypoxia may on cerebral functioning is unknown. On the other hand, ACE2 and its receptors are also found in the brain especially in CNS neurons and glial cells thus making it a potential target for possible direct infiltration by SARS-CoV-2 virus. Individuals infected with SARS-CoV-2 virus have complained of loss of taste and smell with infection of the olfactory bulb suspected to be pathway of entry into the cerebral structures. Since the medical management of COVID-19 is improving with ventilation, corticosteroids and other supportive measures, a large proportion of these patients is expected to recover from the disease. For patients who recover, the possibility of lingering cognitive and behavioural effects are unknown although they are likely to be present. Therefore, the post-acute and long-term effects of COVID-19 on the cognitive and behavioural functioning of individuals are expected to constitute an important area of neuropsychological research and clinical practice in coming months and years.

Materials and Methods. As cases of COVID-19 increase, and with decreasing medical management burden in the post-acute and chronic phase of treatment, patients will likely form a large pool of surviving cases that require neuropsychological assessment and treatment and possibly, long-term rehabilitation. These interventions will be dependent on individual recovery outcomes from the disease. Hence, using Luria's Neuropsychological Assessment methods [4] and those using this framework [5] forms an appropriate serial monitoring method of cognitive and behavioural progress after COVID-19 infection. Therefore, forming a case series of COVID-19 patients from various medical clinics (using multisite protocols) is an appropriate clinical research protocol that should be urgently initiated.

Results. The investigation of individual cases of patients who recovered from COVID-19 should be analyzed individually to portray the sociocultural impacts of the disease and, from a group perspective, general 
trends of cognitive and behavioural trajectories post-recovery may be tracked.

Conclusions. Using Luria's Syndrome Analysis $[4,5]$ to study the neuropsychological profiles of individual COVID-19 patients, ranging in age from infancy to late adulthood, affords a unique opportunity to track the neuropsychological changes of patients recovering from the disease, identify areas of the brain that are susceptible to hypoxic brain damage, predict their outcomes and contribute to the development of future neuropsychological rehabilitation and medical control of long-term sequelae of future viral pandemics impacting the brain.

1. Hosier Hillary, Farhadian Shelli F. and others SARS-CoV-2 infection of the placenta // J Clin Invest. 2020. № 130(9). P. 4947-4953.

2. Fitsiori A., Pugin D., Thieffry C., Lalive P. and Vargas M. I. Unusual Microbleeds in Brain MRI of Covid-19 Patients // Journal of Neuroimaging. 2020. DOI: $10.1111 /$ jon. 12755

3. Li W., Moore M. J., Vasilieva N., Sui J. and others Angiotensin-converting enzyme 2 is a functional receptor for the SARS coronavirus // Nature. 2003. № 426. P. 450-454.

4. Luria A. Higher cortical functions in man. New York: Basic Books, 1966.

5. Glozman J.M. Quantitative and Qualitative Integration of Lurian Procedures // Neuropsychological Review. 1999. № 9. P. 23-32. 